\title{
Vanishing lung syndrome
}

\section{Yu-Tzu Tsao MD, Shih-Wei Lee MD}

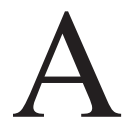
44-year-old man with a 20 pack-year history of smoking presented to the emergency department following a few hours of chest tightness, shortness of breath and cold sweats. He was tachypneic and had no breath sounds over his right hemithorax, which was hyperresonant to percussion. Arterial blood gas analysis on ambient air showed $\mathrm{pH}$ level 7.32 (normal 7.35-7.45), partial pressure of oxygen 74.5 (normal 80-105) $\mathrm{mm} \mathrm{Hg}$, partial pressure of carbon dioxide 32.4 (normal 35-45) mm Hg, bicarbonate level 22.6 (normal 22-32) $\mathrm{mmol} / \mathrm{L}$ and oxygen saturation 92\% (normal $>92 \%$ ). Chest radiography (Figure 1) was interpreted as showing a hyperlucent right hemithorax with a left shift of the mediastinum and a collapsed right lung, which we initially thought was due to a tension pneumothorax. Unexpectedly, chest computed tomography (CT) showed a left-sided pneumothorax with giant bullae on the right (Appendix 1, available at www.cmaj.ca/lookup /suppl/doi:10.1503/cmaj.111507///DC1). Tube thoracostomy re-expanded the left lung, with complete resolution of the patient's symptoms.

Although prevalence estimates are not available, unilateral hyperlucent lung is not an uncommon radiologic finding. ${ }^{1}$ Abnormalities of the thoracic wall and soft tissue are the most common causes. Pneumothorax, emphysema, large bullae, airway obstruction, contralateral pleural effusion and radiographic artifact can also give rise to increased transradiancy of the lung.

Vanishing lung syndrome, also known as type I bullous disease or primary bullous disease of the lung, is defined as large bullae involving at least one-third of a hemithorax. ${ }^{2}$ It usually occurs in young men with a history of smoking and is mainly characterized by giant bullous emphysema in the upper lobes. Its progressive nature may cause exercise intolerance, worsening dyspnea and, occasionally, spontaneous pneumo thorax. ${ }^{3}$ Surgical resection is the treatment of choice, and the best results are seen following limited bullectomy. ${ }^{4.5}$

If it is not possible to see the entire lung edge

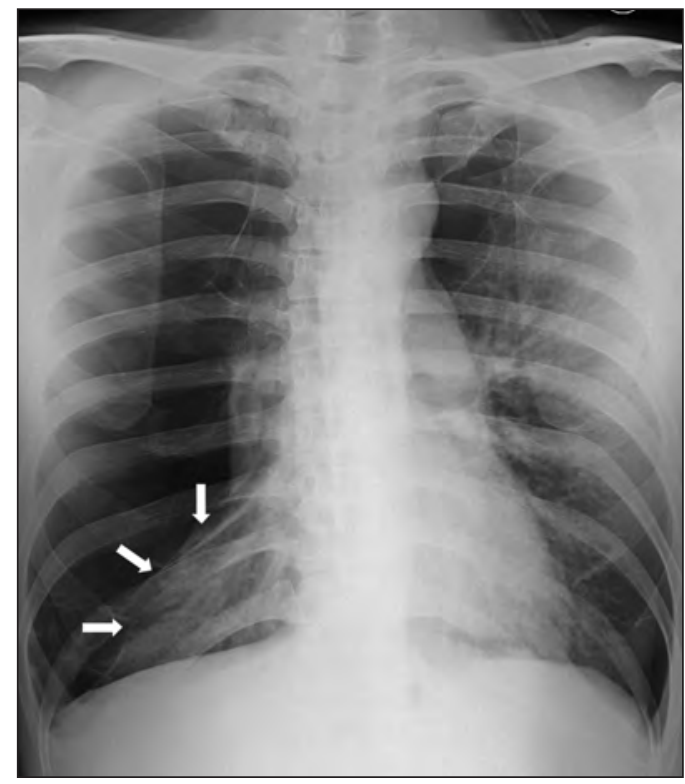

Figure 1: Chest radiograph in a 44-year-old man with chest tightness and shortness of breath, interpreted as showing a hyperlucent right hemithorax with a left shift of the mediastinum and a collapsed lung (arrows).

separated from the chest wall, lung hyperlucency is unlikely to be caused by a straightforward pneumothorax, and CT is needed to evaluate the pleural space and the lung. The unintentional placement of a chest tube into a giant bulla can cause iatrogenic pneumothorax, hemothorax, hemorrhagic shock or death. ${ }^{5}$

\section{References}

1. Dillman JR, Sanchez R, Ladino-Torres MF, et al. Expanding upon the unilateral hyperlucent hemithorax in children. Radiographics 2011;31:723-41.

2. Stern EJ, Webb WR, Weinacker A, et al. Idiopathic giant bullous emphysema (vanishing lung syndrome): imaging findings in nine patients. AJR Am J Roentgenol 1994;162:279-82.

3. Sharma N, Justaniah AM, Kanne JP, et al. Vanishing lung syndrome (giant bullous emphysema): CT findings in 7 patients and a literature review. J Thorac Imaging 2009;24:227-30.

4. Palla A, Desideri M, Rossi G, et al. Elective surgery for giant bullous emphysema: a 5-year clinical and functional follow-up. Chest 2005;128:2043-50.

5. MacDuff A, Arnold A, Harvey J. Management of spontaneous pneumothorax: British Thoracic Society Pleural Disease Guideline 2010. Thorax 2010;65:ii18-31.
Competing interests: None declared.

This article has been peer reviewed.

Affiliations: From the Department of Medicine, Taoyuan General Hospital, Taoyuan, Taiwan

Correspondence to: Shih-Wei Lee, ccm.tygh@gmail.com

CMAJ 2012. DOI:10.1503 /cmaj.111507 